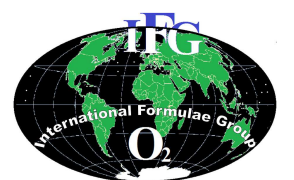

Available online at http://ajol.info/index.php/ijbcs

\title{
Etude de l'activité antihypertensive d'une association de plantes (Schrankia leptocarpa, Garcinia kola et Ocimum americanum) chez le rat Wistar
}

\author{
Coffi T. QUENUM ${ }^{1}$, Hyacinthe AHISSOU ${ }^{2}$, Polycarpe GOUTHON ${ }^{1 *}$ et \\ Anatole LALEYE ${ }^{3}$ \\ ${ }^{1}$ Laboratoire des APS et Motricité, Institut National de la Jeunesse, de l'Education Physique et du Sport \\ (INJEPS), Université d'Abomey-Calavi (UAC), 01 BP : 169 Porto-Novo, Bénin. \\ ${ }^{2}$ Laboratoire d'Enzymologie et Biochimie des Protéines de la Faculté des Sciences et Techniques de \\ l'Université d'Abomey-Calavi (UAC), 01BP : 188 Cotonou, Bénin. \\ ${ }^{3}$ Unité de Biologie Humaine, Faculté des Sciences de la Santé (FSS/ISBA), \\ Université d'Abomey-Calavi (UAC), 01 BP : 188 Cotonou, Bénin. \\ *Auteur correspondant ; E-mail : goupoly@yahoo.fr; Tél : (+229) 90039870.
}

\section{RESUME}

En pharmacopée traditionnelle, le traitement de l'hypertension artérielle (HTA) fait souvent appel à des associations de plantes. L'objectif de cette étude est d'évaluer in vitro et in vivo, l'effet anti-hypertensif de l'association Schrankia leptocarpa, Garcinia kola et Ocimum americanum. L'analyse phytochimique puis l'évaluation de la toxicité ont été réalisées respectivement par la technique de caractérisation en tube, sur les larves de crevette Artemia salina et des rats de souche Wistar. L'effet de l'extrait aqueux de cette association de plantes a été apprécié sur des rats Wistar rendus hypertendus par l'administration de L-Name $(\mathrm{N}(\omega)$-NitroL-Arginine-Méthyl Ester) à la dose de $20 \mathrm{mg} / \mathrm{kg}$ de poids corporel. Un traitement d'une semaine à cet extrait aqueux à dose minimale $(250 \mathrm{mg} / \mathrm{kg}$ de poids corporel), a entraîné une baisse de la pression artérielle (PA) moyenne $(135,8 \pm 12,1 \mathrm{~mm} \mathrm{Hg})$, proche de la normale $(132,3 \pm 5,5 \mathrm{~mm} \mathrm{Hg})$. A la dose maximale de 500 $\mathrm{mg} / \mathrm{kg}$ de poids corporel, la PA passe en dessous de la normale $(116,6 \pm 52,6 \mathrm{~mm} \mathrm{Hg})$, sans toutefois qu'il y ait une différence significative entre les deux doses $(\mathrm{p}>0,05)$. Les molécules des groupes chimiques présents dans l'extrait aqueux interviendraient donc dans la régulation des paramètres cardiovasculaires. L'association de plantes étudiée a un effet positif sur l'HTA induite et les résultats semblent indiquer son innocuité.

(C) 2014 International Formulae Group. All rights reserved.

Mots clés : Pression artérielle, plantes médicinales, hypotenseur, toxicité.

\section{INTRODUCTION}

L'hypertension artérielle est un problème de santé publique croissant dans les pays en développement, où le mode d'alimentation à l' "occidental » se propage et remplace souvent celui traditionnel (OMS, 2002). En Afrique, plus de 20 millions de personnes souffrent d'hypertension artérielle; sa prévalence est comprise entre 25 et $35 \%$ chez les adultes de 25 à 64 ans avec une tendance à l'aggravation avec le temps (OMS, 2006; Fakher et al., 2006). Au Bénin, les données épidémiologiques montrent que la prévalence de l' HTA est passée de $13,6 \%$ en 2001 à $20,2 \%$ en 2004 et à $27,9 \%$ en 2008 (Houinato et al., 2008 ; OMS, 2008). 
Le traitement de l'HTA fait appel à diverses molécules de synthèse mais de plus en plus les populations des pays en voie de développement se tournent vers la pharmacopée traditionnelle. Par ailleurs, outre l'adoption d'une bonne hygiène alimentaire, plusieurs études ont montré que la pratique régulière d'activités physiques même modérée permet d'améliorer la circulation sanguine, de protéger les artères et d'abaisser la pression artérielle (Poirier et Després, 2003).

La thérapeutique par les plantes, cette pratique ancestrale, connaît depuis quelques années un regain d'intérêt en raison de la diminution $\mathrm{du}$ pouvoir d'achat des populations, du coût élevé des médicaments conventionnels et de la crainte des produits de synthèse (Perroti, 1999). Comme cela a été indiqué pour la Médecine moderne (Bassand, 2008), les tradipraticiens ont souvent recours à cette même stratégie dans le traitement de l'HTA. Dans le département de l'Ouémé au sud du Bénin, une association de plantes faites de Schrankia leptocarpa, Garcinia kola et Ocimum americanum est particulièrement utilisée dans la lutte contre l'hypertension artérielle. Cette thérapeutique est administrée de façon empirique sans aucune connaissance réelle de la toxicité et des effets biologiques connexes de ces plantes. Aussi, nous proposons-nous de rechercher les effets anti hypertensifs de cette association de plantes utilisées au sud du Bénin afin de confirmer ou d'infirmer leurs actions thérapeutiques sur l'hypertension artérielle.

\section{MATERIEL ET METHODES}

\section{Matériel}

\section{Matériel végétal}

Le matériel végétal était constitué de parties aériennes d'Ocimum americanum, de Schrankia leptocarpa et des noix de Garcinia Kola récoltées en octobre 2012 à Porto-Novo, identifiées à l'Herbier National du Bénin ( $\mathrm{N}^{\circ}$ AA $6431 / \mathrm{HNB}$ et $\mathrm{N}^{\circ} \mathrm{AA} 6432 / \mathrm{HNB}$.). Les plantes ont été séchées à l'abri de la lumière pendant 14 jours puis moulues. Les noix de Garcinia kola ont été aussi réduites en poudre puis mélangées à celle des plantes à proportions égales soit $150 \mathrm{~g}$ de poudre par plante.

\section{Matériel animal}

Le matériel animal était constitué de 55 rats de souche Wistar et des larves de crevettes Artemia salina.

Les rats étaient gardés dans les conditions d'élevage de l'animalerie de l'Unité de Biologie Humaine de la FSS de l'Université d'Abomey-Calavi. Ils avaient libre accès à l'eau et à la nourriture avec un cycle de 12 heures de lumière et $12 \mathrm{~h}$ d'obscurité. Leur poids moyen était de $162 \pm$ 5,57 g pour l'étude de la toxicité aigüe in vivo et de $203 \pm 21,63 \mathrm{~g}$ pour l'étude de l'effet de l'extrait aqueux. Les larves de crevettes Artemia salina ont servi à l'étude de la toxicité larvaire (toxicité in vitro).

\section{Méthodes}

\section{Tests de toxicité}

- Test préliminaire de toxicité non clinique : les larves de crevette étaient obtenues après incubation et sous agitation douce pendant quarante-huit heures d'œufs d'Artemia salina à la température du laboratoire $\left(28{ }^{\circ} \mathrm{C}-30\right.$ ${ }^{\circ} \mathrm{C}$ ) dans un erlenmeyer de $1000 \mathrm{ml}$, contenant de l'eau de mer prélevée dans l'océan Atlantique à Cotonou.

Une solution mère de l'extrait aqueux est préparée dans l'eau de mer à une concentration de $50 \mathrm{mg} / \mathrm{ml}$. Puis dans dix tubes à essai, une gamme de concentrations décroissantes de l'extrait a été réalisée, en procédant à une dilution en série de raison deux de la solution mère (soit $25 \mathrm{mg} / \mathrm{ml}$ dans le premier tube et $0,05 \mathrm{mg} / \mathrm{ml}$ dans le dernier). Dans chaque tube ainsi préparé, il est alors ensemencé 16 larves contenues dans $1 \mathrm{ml}$ d'eau de mer. L'ensemble a été incubé pendant 24 heures à la température du 
laboratoire, c'est-à-dire $28-30{ }^{\circ} \mathrm{C}$. Après 24 heures, les tubes à essai ont été examinés et le nombre de survivants dans chacun a été compté et enregistré. Les larves ont été considérées comme mortes, si elles ne présentent pas de mouvement interne ou externe pendant 10 secondes d'observation. Les larves étaient maintenues à jeun pendant l'expérimentation. Pour s'assurer que la mort observée dans les tubes à essais est attribuée uniquement aux extraits et non à la faim, on compare les tubes expérimentaux contenant l'extrait à des tubes témoins contenant des solutions larvaires uniquement. Les larves de crevette Artemia peuvent survivre jusqu'à 48 heures sans s'alimenter (Michael et al., 1956; Harwig et al., 1997) du fait qu'elles se nourrissent de leur sac vitellin (Migliore et al., 1997).

- Test de toxicité aigue in vivo : l'étude de toxicité sur les animaux a été réalisée selon les recommandations de l'Organisation de Coopération et de Développement Economique (OCDE, 2001). Six rats de souche Wistar, femelles nullipares et non gravides répartis en deux lots ont été utilisés pour cette étude; un groupe contrôle était constitué de sept rats. Au premier lot de trois rats, il a été administré l'extrait à la dose de $300 \mathrm{mg} / \mathrm{kg}$ de poids corporel puis au second lot la dose limite de $2000 \mathrm{mg} / \mathrm{kg}$ de poids corporel. Un suivi sur 14 jours après administration a permis de rechercher d'éventuels signes cliniques de morbidité ou de mortalité et d'apprécier l'évolution du poids corporel. Au jour J14, des dosages biochimiques $\left(\mathrm{Na}^{+}, \mathrm{Cl}^{-}, \mathrm{K}^{+}\right.$, ASAT, ALAT, Glycémie, Créatininémie, Cholestérol total) et l'étude histologique des reins et du foie ont été réalisés après sacrifice des animaux.

\section{Traitements}

Au premier Jour (J0), les rats ont été pesés et répartis en six lots (G1 à G6) de sept animaux: le lot G1 est celui des rats contrôle (témoin négatif) n'ayant subi aucune induction et cinq lots, de G2 à G6, ayant reçu par gavage du L-Name (N( $\omega)$-Nitro-LArginine-Méthyl Ester) à la dose unique journalière de $20 \mathrm{mg} / \mathrm{kg}$ de poids corporel pendant sept jours.

$\mathrm{Au}$ huitième jour, après une nouvelle pesée des animaux, ceux des lots G2 à G5 ont été soumis pendant sept jours respectivement aux traitements suivants :

- lot G2: rats sacrifiés au $8^{\text {ème }}$ jour c'est-àdire à la fin de l'induction de l'HTA par l'administration de L-Name.

- lot G3 : rats n'ayant reçu aucun traitement (témoin positif),

- lot G4 : rats traités à l'extrait aqueux à la dose de $250 \mathrm{mg} / \mathrm{kg}$ de poids corporel/jour;

- lot G5: rats traités à l'extrait aqueux à la dose de $500 \mathrm{mg} / \mathrm{kg}$ de poids corporel/jour ;

- lot G6 : rats traités au Captopril $^{\circledR}$, un inhibiteur de l'enzyme de conversion de l'angiotensine à la dose de $100 \mathrm{mg} / \mathrm{kg}$ de poids corporel/jour.

$\mathrm{Au}$ lendemain de la fin des différents traitements, c'est-à-dire à J15, les masses corporelles des rats ont été relevées une dernière fois (Tableau 1) ainsi que leurs pressions artérielles (Tableau 2). Le dispositif expérimental utilisé pour l'enregistrement de la pression artérielle est un moniteur de marque SpaceLabs. Le rat a été anesthésié par injection intrapéritonéale de thiopental à la dose de $40 \mathrm{mg} / \mathrm{kg}$ de poids corporel. Sa carotide a été disséquée et intubée à l'aide d'un cathéter relié au manomètre qui intègre les variations de la pression carotidienne. De plus les différents organes tels que le foie, les reins et l'estomac ont été prélevés aux fins d'études histologiques. L'observation des coupes histologiques a été faite sur un microscope photonique de marque Olympus BX 60 couplé à un ordinateur.

\section{Analyse statistique}

Les données enregistrées ont été traités à l'aide du logiciel Statistica Version 7.1 de 
STAT Soft Inc. Pour chaque variable, la moyenne (m) et l'erreur standard de la moyenne (SEM) ont été calculées. Les résultats des différents groupes ont été comparés en utilisant l'analyse de variance (ANOVA) de Kruskal Wallis et le test U de Mann Whitney. Le test de rang de Wilcoxon a été utilisé pour les comparaisons intragroupes. Le niveau de signification statistique des résultats a été fixé à $\mathrm{p}<0,05$.

\section{RESULTATS}

\section{Analyse phytochimique}

Des tanins galliques, des flavonoïdes, des dérivés quinoniques, des mucilages et des composés réducteurs constituent les grands groupes chimiques présents dans cette association de plantes.

\section{Etude de la toxicité}

\section{Test de toxicité larvaire}

La lecture de la courbe permet de déduire que la mortalité des larves respecte une relation de dose-réponse du fait que le nombre de larves mortes augmente quand la concentration augmente au moyen de l'ajustement logarithmique présenté sur la Figure 1, la concentration qui cause la mort de la moitié des 16 larves introduites $\left(\mathrm{CL}_{50}\right)$ correspond à une valeur de $0,63 \mathrm{mg} / \mathrm{ml}$.

\section{Tests de toxicité aigue chez le rat}

De rares effets indésirables et un cas de décès d'animaux a été enregistré durant les 14 jours d'observation clinique. Il est mort au cours de la période de traitement (c'est-à-dire après J7) et un autre a survécu mais avec une paralysie des membres antérieurs.

Une légère augmentation de la masse corporelle des rats est observée quelle que soit la dose de l'extrait tout au long de l'expérimentation $(\mathrm{p}=0,06)$. Le Tableau 3 récapitule les valeurs des paramètres biochimiques obtenus aussi bien chez les rats traités que chez les rats témoins.
La glycémie paraît plus élevée pour les deux doses utilisées par rapport au groupe témoin avec une différence significative $\mathrm{p}=0,004$, alors que les valeurs de la créatininémie et du potassium ont diminué de façon significative par rapport au groupe témoin à $\mathrm{p}=0,004$. Quant au cholestérol total, une diminution insensible a été observée (Tableau 3). Une différence significative a été également enregistrée entre la dose minimale et maximale en ce qui concerne la créatininémie, le potassium et le chlore (Tableau 3). Cependant, nous notons une différence significative entre les deux doses (minimale et maximale).

Les différentes coupes histologiques observées (estomac, foie et rein) nous montrent des structures parfaitement normales (Figures 4, 5 et 6 ).

\section{Etude des propriétés anti-hypertensives de l'extrait}

Les chiffres tensionnels des rats traités par l'extrait aqueux à la dose maximale (500 $\mathrm{mg} / \mathrm{kg}$ de poids corporel) sont plus bas que ceux des rats traités au Captopril ${ }^{\circledR}(116,6 \pm$ $52,6 \mathrm{~mm} \mathrm{Hg}$ versus $118,7 \pm 53,7 \mathrm{~mm} \mathrm{Hg}$ ), qui par ailleurs sont plus bas que ceux des rats témoins $(118,7 \pm 53,7 \mathrm{~mm} \mathrm{Hg}$ versus $124,6 \pm$ $55,7 \mathrm{~mm} \mathrm{Hg})$.

Le Tableau 3 indique qu'à la dose minimale $(250 \mathrm{mg} / \mathrm{kg}$ de poids corporel), la pression artérielle moyenne des rats diminue avoisinant presque la normale $(135,3 \mathrm{~mm} \mathrm{Hg}$ versus $132,3 \pm 5,5 \mathrm{~mm} \mathrm{Hg}$ ); à la dose maximale de $500 \mathrm{mg} / \mathrm{kg}$ de poids corporel, la pression artérielle moyenne tend vers une hypotension (116,3 $\mathrm{mm} \mathrm{Hg}$ versus $132,3 \pm 5,5$ $\mathrm{mm} \mathrm{Hg).}$ 
Tableau 1 : Caractéristiques biométriques des rats.

\begin{tabular}{|c|c|c|c|c|c|}
\hline & $\begin{array}{c}\text { Rats } T \text { positif } \\
\text { (G3) }\end{array}$ & $\begin{array}{c}\text { Extrait aqueux } \\
\max (\text { G5) }\end{array}$ & $\begin{array}{c}\text { Captopril }^{\circledR} \\
\text { (G6) }\end{array}$ & $\begin{array}{r}\text { HTA } \\
\text { (G2) }\end{array}$ & $\begin{array}{l}\text { Contrôle } \\
\text { (G1) }\end{array}$ \\
\hline & $\mathrm{m} \pm \mathrm{SEM}$ & $\mathbf{m} \pm \mathrm{SEM}$ & $\mathbf{m} \pm \mathrm{SEM}$ & $\mathbf{m} \pm$ SEM & $\mathbf{m} \pm$ SEM \\
\hline MCJO (g) & $200,1 \pm 18,9$ & $214,0 \pm 24,7$ & $195,1 \pm 19,1$ & $196,6 \pm 10,9$ & $190,6 \pm 10,9$ \\
\hline MCJ7 (g) & $198,4 \pm 18,0$ & $204,7 \pm 22,2$ & $197,4 \pm 19,8$ & $205,2 \pm 10,2$ & $188,2 \pm 10,7$ \\
\hline MCJ14 (g) & $211,3 \pm 19,4$ & $224,3 \pm 22,1$ & $197,0 \pm 19,5$ & - & $187,4 \pm 8,3$ \\
\hline
\end{tabular}

Rats T positif : rats du groupe des témoins positifs ; Extrait aqueux max : extrait aqueux à la dose maximale ; HTA : hypertension artérielle; m : moyenne ; SEM : erreur standard de la moyenne ; MC : masse corporelle ; J0 : $1^{\text {er }}$ jour d'induction; J7 : $7^{\text {ème }}$ jour d'induction; J14 : $14^{\text {ème }}$ jour d'induction.

Tableau 2 : Caractéristiques de la pression artérielle des rats de souche Wistar de l'étude.

\begin{tabular}{|c|c|c|c|c|c|}
\hline $\begin{array}{l}\text { Pression } \\
\text { artérielle }\end{array}$ & $\begin{array}{c}\text { Rats T } \\
\text { positifs (G3) }\end{array}$ & $\begin{array}{c}\text { Extrait } \\
\text { aqueux (G5) }\end{array}$ & $\begin{array}{c}\text { Captopril@ } \\
\text { (G6) }\end{array}$ & HTA (G2) & $\begin{array}{c}\text { Contrôle } \\
\text { (G1) }\end{array}$ \\
\hline & $\mathrm{m} \pm \mathrm{SEM}$ & $\mathrm{m} \pm \mathrm{SEM}$ & $\mathbf{m} \pm \mathbf{S E M}$ & $\mathrm{m} \pm \mathrm{SEM}$ & $\mathbf{m} \pm \mathrm{SEM}$ \\
\hline PAS (mm Hg) & $162,3 \pm 16,3$ & $149,0 \pm 12,4$ & $153,3 \pm 14,8$ & $176,8 \pm 8,4$ & $145,0 \pm 3,7$ \\
\hline PAD $(\mathrm{mm} \mathrm{Hg})$ & $136,8 \pm 7,9$ & $129,6 \pm 12,7$ & $131,2 \pm 13,6$ & $161,8 \pm 14,9$ & $126,0 \pm 7,3$ \\
\hline PAM (mm Hg) & $124,6 \pm 55,7$ & $116,6 \pm 52,6$ & $118,7 \pm 53,7$ & $166,8 \pm 12,3$ & $132,3 \pm 5,5$ \\
\hline
\end{tabular}

Tableau 3 : Comparaison des valeurs biochimiques entre les groupes G3, G4 et G5.

\begin{tabular}{|c|c|c|c|}
\hline \multirow[t]{2}{*}{ Paramètres } & $\begin{array}{c}\text { Rats } T \text { positifs } \\
\text { (G3) }\end{array}$ & $\begin{array}{c}\text { Dose minimale } \\
\text { (G4) }\end{array}$ & $\begin{array}{c}\text { Dose maximale } \\
\text { (G5) }\end{array}$ \\
\hline & $\mathbf{m} \pm$ SEM & $\mathrm{m} \pm \mathrm{SEM}$ & $\mathrm{m} \pm \mathrm{SEM}$ \\
\hline GLY (g/dL) & $0,83 \pm 0,16$ & $1,49 \pm 0,03 * *$ & $1,37 \pm 0,21 \dagger \dagger \dagger$ \\
\hline CREA (mg/L) & $9,28 \pm 0,95$ & $5,22 \pm 0,38 * *$ & $6,00 \pm 2,00 \dagger \dagger \dagger$ \\
\hline $\mathbf{N a}^{+}(\mathrm{mEq} / \mathrm{L})$ & $141,28 \pm 1,89$ & $142,33 \pm 1,15$ & $140,67 \pm 1,53$ \\
\hline $\mathbf{K}^{+}(\mathrm{mEq} / \mathrm{L})$ & $6,11 \pm 0,67$ & $3,78 \pm 0,61 * *$ & $5,24 \pm 1,34 \dagger \dagger \dagger$ \\
\hline $\mathrm{Cl}^{-}(\mathrm{mEq} / \mathrm{L})$ & $98,57 \pm 1,81$ & $105,33 \pm 1,15^{* *}$ & $104 \pm 2,00 \dagger \dagger \dagger$ \\
\hline CT $(g / L)$ & $0,85 \pm 0,13$ & $0,66 \pm 0,07$ & $0,67 \pm 0,14$ \\
\hline ASAT (UI/L) & $182,66 \pm 31,72$ & $168,00 \pm 6,08$ & $144,66 \pm 51,5$ \\
\hline ALAT (UI/L) & $91,00 \pm 4,58$ & $70,00 \pm 8,72$ & $82,00 \pm 8,88$ \\
\hline
\end{tabular}

Rats $\mathrm{T}$ positifs : rats du groupe des témoins positifs; Dose minimale : extrait aqueux à la dose minimale; Dose maximale : extrait aqueux à la dose maximale ; $\mathrm{m}$ : moyenne ; SEM : erreur standard de la moyenne ; GLY : glycémie ; CREA : créatininémie; $\mathrm{Na}^{+}$: sodium $; \mathrm{K}^{+}$: potassium $; \mathrm{Cl}^{-}$: chlorure $; \mathrm{CT}$ : cholestérol total ; $* *$ : différence entre les groupes G4 et G5, significative à $\mathrm{p}=0,01 ; \dagger \dagger \dagger$ : différence entre G3 et G5, significative à $\mathrm{p}=0,004$. 


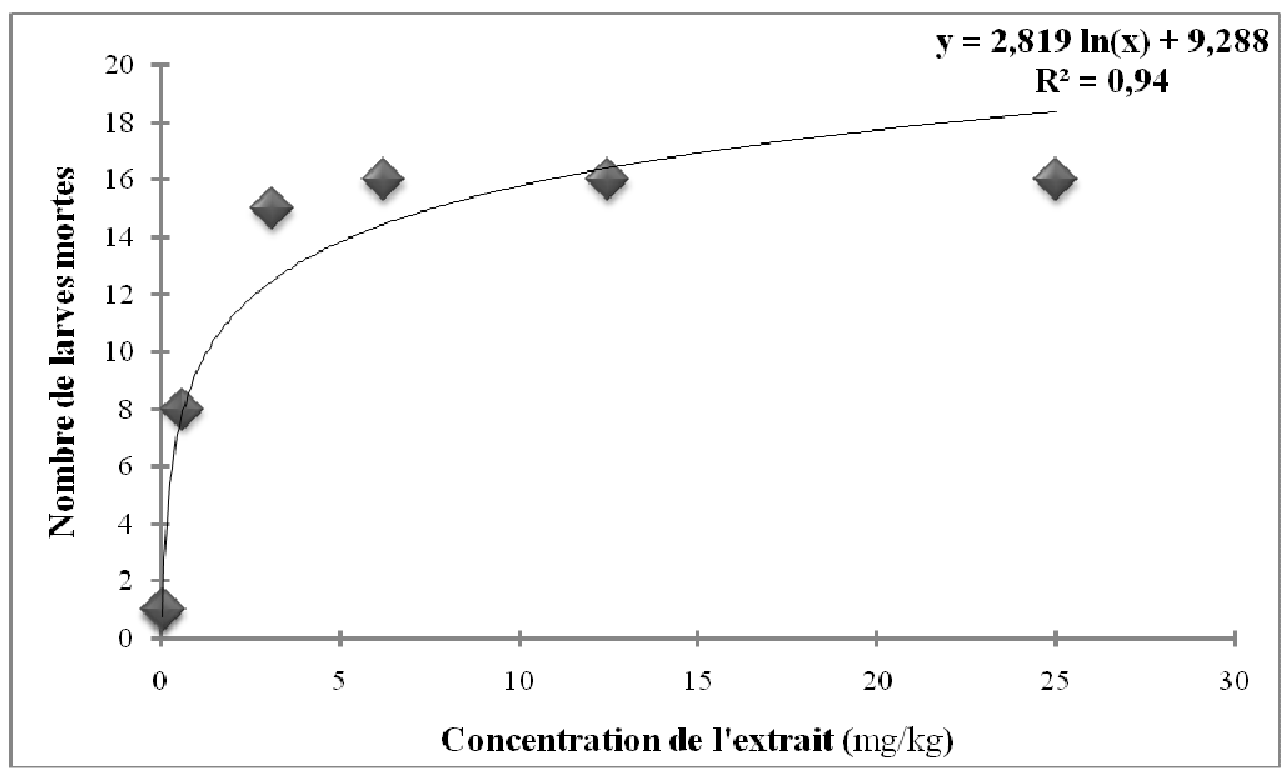

Figure 1: Courbe de variation de la mortalité larvaire en fonction de la concentration de l'extrait aqueux.

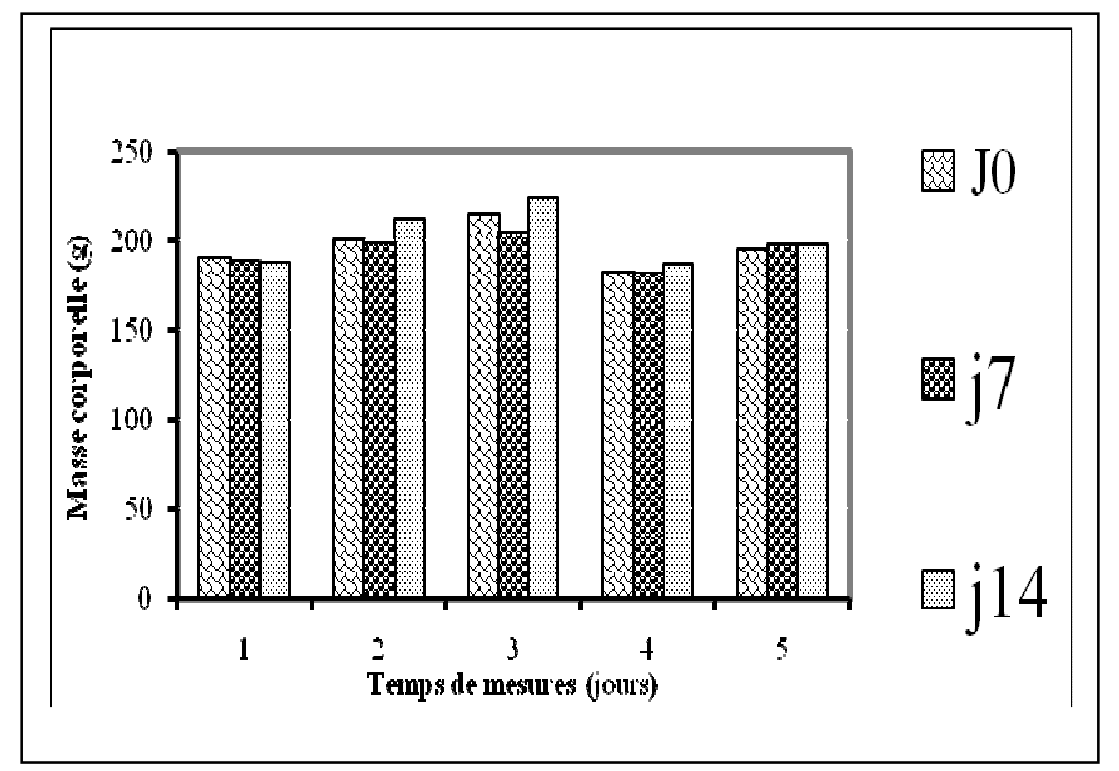

Figure 2 : Evolution de la masse corporelle des rats entre $\mathrm{J} 0$ et $\mathrm{J} 14.1:$ rats sains ; $2:$ témoins ; 3 : extrait aqueux à la dose maximale ( $\dagger \dagger$ : différence entre les masses corporelles de $\mathrm{J} 7$ et $\mathrm{J} 14$, significative à $\mathrm{p}=0,02$; $\dagger$ : différence entre les masses corporelles de $\mathrm{J} 0$ et $\mathrm{J} 14$, significative à $\mathrm{p}=0,03) ; 4$ : extrait aqueux à la dose minimale ; 5 : Captopril ${ }^{\circledR} \mathrm{J} 0$ : $1^{\mathrm{er}}$ jour de l'induction ; $\mathrm{J} 7:$ septième jours d'induction ; J14 : $14^{\mathrm{ème}}$ jour d'induction. 


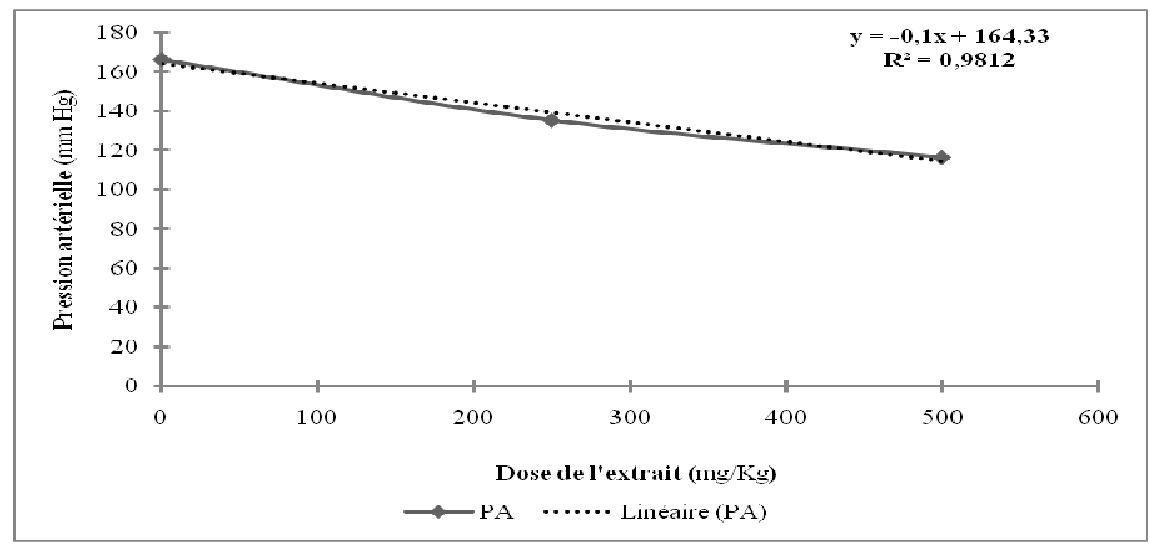

Figure 3 : Evolution de la pression artérielle en fonction de la dose de l'extrait aqueux.

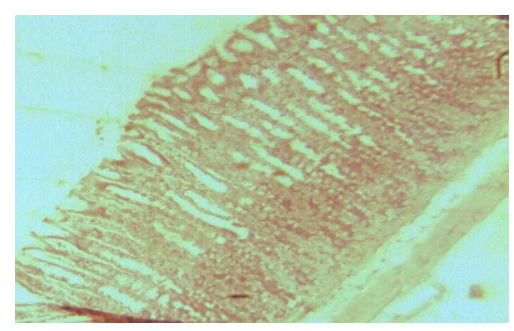

Figure 4 : Estomac témoin: vue d'ensemble de la paroi digestive montrant de gauche à droite l'épithélium gastrique.

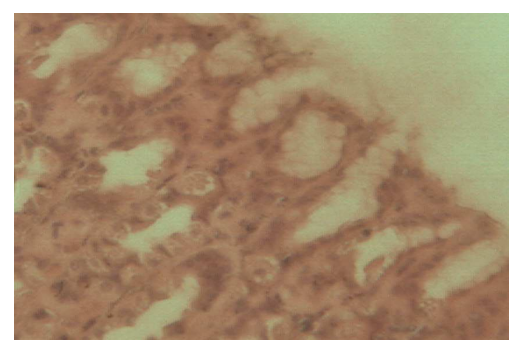

Figure 5 : Estomac témoin montrant un épithélium gastrique normal et des glandes gastriques.

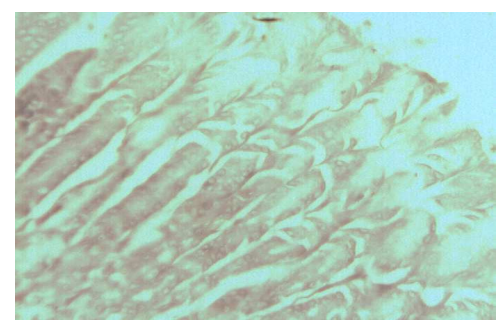

Figure 6: Estomac de rat soumis à un essai limite de toxicité (2000 mg/kg) avec l'extrait aqueux (HE x 10). Les différentes tuniques de la paroi gastrique sont normales. 


\section{DISCUSSION}

A notre connaissance, aucune étude antérieure n'a évalué l'effet biologique de notre association de plantes sur un modèle animal d'HTA. Par contre, des études ont été réalisées sur chacune des plantes de façon séparée. L'analyse phytochimique de l'association des plantes a révélé la présence des tanins, coumarines, flavonoïdes, stéroïdes, mucilages et composés réducteurs. Ces résultats confirment les travaux de Yemoa et al. (2008) qui ont porté sur Garcinia kola et ont noté la présence de tanin et de flavonoïdes. Ocimum canum Sims, encore appelée Ocimum americanum, est une herbacée annuelle, aromatique et endémique de l'Afrique subsaharienne. La chromatographie sur couche mince (CCM) y indique la présence de flavonoïdes et de phénols (Khalil, 2009). Il a des propriétés antimicrobiennes (Yayi-ladekan et al., 2011; Atanasova et al., 2009).

En effet, les tanins interviendraient entre autres dans l'inhibition de certaines enzymes telles que la 5-lipo oxygenase et l'enzyme de conversion de l'angiotensine. Ces molécules pourraient augmenter la biodisponibilité du mono-oxyde d'azote (NO) en apportant un surplus en L-arginine (JianWei et al., 2009). Alors que les flavonoïdes constituent un groupe de métabolites très répandus dans les plantes. Ils se retrouvent dans presque toutes les parties de la plante, à différentes concentrations où ils jouent un rôle déterminant dans le système de défense comme antioxydants (Middleton et al., 2000). Ces différents composés énumérés sont intéressants car associés à de nombreuses activités biologiques: anti-inflammatoires (Middleton et al., 2000), anti-hépatotoxiques, anti-tumorales (Milane, 2004), antihypertensives, anti-thrombiques, antibactériennes, antivirales, antiallergiques et antioxydantes.
Quant aux coumarines simples, elles contribuent généralement à fluidifier le sang par leur activité veinotonique, vasoprotectrice et vasodilatatrice coronarienne. Cette fluidité faciliterait la circulation sanguine donc baisserait la pression au niveau des artères (OMS, 2006).

Les stéroïdes constituent un groupe de lipides dérivant de triterpénoïdes et sont cardiotoniques. Les mucilages quant à eux constituent des polysaccharides. Ces différents groupes chimiques présents dans notre extrait, d'après leurs propriétés interviennent dans la régulation des troubles cardiovasculaires. De récentes études comme celle de Lagnika et al. (2012) indique que Schrankia Leptocarpa possède des propriétés anti-oxydantes et que, le Garcinia kola est utilisé pour le traitement de l'HTA. L'utilisation de cette association de plantes, serait donc justifiée puisque Bassand (2008) a montré qu'il est rare de ramener les chiffres tensionnels à un niveau désiré grâce à une monothérapie conventionnelle. Des associations contenant deux, trois, voire quatre médicaments sont parfois nécessaires.

En se référant à l'échelle de toxicité établie par Mousseux (1995), la valeur $\mathrm{CL}_{50}$ de l'extrait est supérieure à $0,1 \mathrm{mg} / \mathrm{ml}$, valeur au-dessus de laquelle l'extrait est considéré comme ne présentant pas de toxicité. Il ressort donc que l'extrait aqueux de l'étude ne serait pas toxique.

Une diminution significative de la créatinémie a été observée entre les différents groupes et le lot témoin. Ceci traduit son innocuité relative au niveau rénal. Bien qu'il n'y ait pas de différence significative entre les lots de rats G3 et G4 en ce qui concerne la transaminasémie, notons néanmoins que l'activité hépatique était plus importante à la dose minimale (ASAT : 182,66 $\pm 31,72 \mathrm{UI} / \mathrm{L}$; ALAT : $91,00 \pm 4,58 \mathrm{UI} / \mathrm{L})$ qu'à la dose maximale (ASAT: 168,00 \pm 6,08 UI/L ; ALAT : 70,00 $\pm 8,72 \mathrm{UI} / \mathrm{L}$ ). Ceci pourrait indiquer qu'à la dose maximale, l'extrait et 
principalement Ocimum americanum aurait révélé ses propriétés hépato protectrices (Milane, 2004 ; Sharma et al., 2012). Ceci conforte les résultats de l'étude histologique du foie. L'augmentation de la glycémie paraît surprenante d'autant plus que le Garcinia kola devrait favoriser sa diminution. (Adaramoye, 2012). Une attention particulière devrait donc être accordée à la surveillance de la glycémie au cours du traitement.

La baisse non significative du taux cholestérol total suggère que l'extrait aqueux étudié n'aurait pas d'effet sur la lipidémie et ne contribuerait donc pas à l'installation de pathologies de surcharge pondérale et leurs corollaires.

L'extrait étudié réduit la pression artérielle induite par le L-NAME de façon plus remarquable que la drogue de référence qu'est le Captopril $^{\circledR}$. Il lèverait donc l'inhibition de la synthèse du NO (puissant vasodilatateur) provoquée par le L-NAME. Son mécanisme d'action pourrait être un relâchement de la musculature lisse vasculaire conduisant à une diminution des résistances périphériques. Quant à l'efficacité de l'extrait, deux doses respectives ont été utilisées (250 et $500 \mathrm{mg} / \mathrm{kg}$ de poids corporel). La dose minimale ayant ramené la forte pression artérielle observée au huitième jour à presque la normale, nous en déduisons que l'extrait aqueux étudié aurait une action positive sur l'hypertension artérielle et cette dose pourrait être considérée comme dose minimale efficace de cet extrait.

Le pouvoir antihypertenseur de l'extrait serait principalement dû à la présence des flavonoïdes, des tanins et des coumarines qui sont des composés phénoliques dont le pouvoir antioxydant et anti radicalaire sont bien connus.

\section{Conclusion}

Cette étude avait pour objectif de déterminer in vitro et in vivo, l'effet anti- hypertensif de l'association Schrankia leptocarpa, Garcinia kola et Ocimum americanum. Les groupes chimiques présents dans l'extrait aqueux interviennent dans la régulation des paramètres cardiovasculaires. Le traitement par l'extrait aqueux qui a suivi l'induction de l'HTA chez les rats de souche Wistar met en évidence une amélioration des chiffres tensionnels par rapport aux témoins positifs et même plus que ceux ayant été traités au Captopril ${ }^{\circledR}$ (drogue de référence), ce qui témoigne de l'efficacité de l'extrait. L'association de plantes étudiée a donc une action positive sur l'hypertension artérielle et son innocuité a été prouvée. Tous ces résultats soutiennent la phytothérapie. Pour que la médecine traditionnelle soit viable, il faut s'efforcer de préserver tant les populations végétales que les connaissances relatives à leur utilisation à des fins médicales.

\section{REMERCIEMENTS}

Les auteurs remercient les responsables $\mathrm{du}$ centre d'entretien musculaire et cardiovasculaire VITA FORME de PortoNovo, pour leur assistance technique lors de la recherche documentaire et du traitement statistique des données. Nous remercions également le Professeur Gbénou Joachim qui a bien voulu accepter que les analyses phytochimiques soient réalisées dans le Laboratoire de Pharmacognosie et des Huiles Essentielles (FAST/Université d'AbomeyCalavi) qu'il dirige.

\section{REFERENCES}

Adaramoye O. 2012. Antidiabetic effect of kolaviron, a biflavonoid complex isolated from Garcinia kola seeds, in Wistar rats. Afr. Health Sci., 12(4): 498-506.

Adrogué H, Madias N. 2013. Sodium surfeit and potassium deficit: Keys to the pathogenesis of hypertension. J. Am. Soc. Hypertens., 4(13): 169-171. 
Anderson C, Hallberg A, Hogberg T. 1996.Advances in the development of pharmaceutical antioxidants. Adv. Drug Res., 28(5): 65-180.

Atanasova M, Ribarova F. 2009. Phenols et flavonoides totaux dans les extraits secs des feuilles des bouleaux argentes bulgares (Betula pendula). Rev. Gén. Industr., 4: 21-25.

Bassand JP. 2008. Prévention des maladies cardiovasculaires. Besançon Cardio. [En ligne]. Juin [17/03/13]. Disponible à l'URL : http:// www.besancon-cardio.org.

Cheville A, Dose A, Basford J, Rhudy L. 2012. Insights into the reluctance of patients with late-stage cancer to adopt exercise as a means to reduce their symptoms and improve their function. $J$. Pain. Symptom. Manage., 44(1): 84-94.

Diallo A. 2005. Etude de la phytochimie et des activités biologiques de Syzynum guinense WILLD (Myrtaceae). Thèse de Pharmacie, Université de Bamako, Bamako, p. 99.

Duarte J, Jimenez R, O'valle F, Galisteo M. 2002. Protective effects of the flavonoid quercetin in chronic nitric oxide deficient rats. J. Hypertens., 20(4): 1843-1854.

Fakher N, Nathalie S, Chahwakilian A, Sylvie D-M, Safar M, Blacher J. 2006. Antihypertenseurs : pour une meilleure efficacité chez le sujet âgé. Med. Ther. Cardio., 2(5): 506-512.

Ghannem H, Hadj F. 1997. Transition épidémiologique et facteurs de risque cardiovasculaire en Tunisie. Rev. Epidé. Santé Publique, 45(4): 286-292.

Houinato D, Gbary A, Houéhanou Y, Djrolo F, Amoussou M, Segnon-Agueh J et al. 2008. Prévalence de l'hypertension artérielle et facteurs de risque associés en population générale au Bénin. Rev. Epidémiol. Santé Publique., 60(2): 95102.
Jian-Wei G, Manning D, Young E, Shparago M, Sartin B, Bailey A. 2009. Nitric oxide synthesis inhibition in harlanspraguedawley rats: a model of malignant hypertension. Am. J. Physiol. Regul. Integr. Comp. Physiol., 297(1): 142-148.

Khan K, Thompson A, Blair S, Sallis J, Powell K, Bull F, Bauman A. 2012. Sport and exercise as contributors to the health of nations. The Lancet., 380(9836): 5964.

Lagnika L, Prodjinonto U, Attioua B, Sanni A.2012. Chemical analysis, antimicrobial and antioxidant activities of eight extracts from Schrankia leptocarpa L. Afri. J. Biotech., 11(72), 13739-13745.

Middleton E, Kandaswami C, Theoharides T. 2000. The effects of plant flavonoids on mammalian cells: implications for inflammation, heart disease and cancer. Pharmacol. Rev., 52(21): 673-751.

Milane H. 2004. La quercétine et ses dérives : molécules à caractère pro oxydant ou capteurs de radicaux libres; études et applications thérapeutiques. Thèse, Université Louis Pasteur, Paris, p. 13-36.

Mousseux M.1995. Test de toxicité sur les larves d'artémia salina entretien d'un élevage de balanes. Université française de Pacifique. Centre universitaire de Nouvelle Calédonie. Deust. Aquaculture, p. 20.

OCDE (Organisation de Coopération et Développement Economique). 2001. Lignes directrices pour les essais de produits chimiques (section 4 : effets sur la santé; essai $\mathrm{n}^{\circ} 423$ : toxicité orale aigue; méthode par classe de toxicité adopté le 17 décembre, OCDE.

OMS (Organisation Mondiale de la Santé). 2006 . Éviter une Crise Cardiaque ou un Accident Vasculaire Cérébral. OMS : 20 Avenue Appia, 1211 Genève 27.

OMS (Organisation Mondiale de la Santé). 2002. Alimentation et Santé Publique : un 
Constat Inquiétant. OMS : 20 Avenue Appia, 1211 Genève 27.

OMS (Organisation Mondiale de la Santé). 2008. Rapport final de l'enquête STEPS au Bénin, Cotonou. Ministère de la Santé, Direction Nationale de la Protection Sanitaire, Programme National de Lutte contre les Maladies Non Transmissibles. OMS, 20 Avenue Appia, 1211 Genève 27 ; 101p.

Perroti C. 1999. Effet des plantes médicinales sur les maladies cardiovasculaires. Phytothérapie., p. 1-90.

Poirier P, Després J-P. 2003. Obésité et Maladies Cardiovasculaires. Med Sci : Paris ; 943-949.

Pelka M, Danzl C, Distler W, Petschelt A. 2000. A new screening test toxicity testing of dental materials. J. Dent., 28: 341-345.

Sciolino N, Holmes P, Natale R. 2012. Exercise offers anxiolytic potential: A role for stress and brain noradrenergicgalaninergic mechanisms. Neurosci. Biobehav. Rev., 36(9): 1965-1984.

Sharma J, Gairola S, Gaur R, Painuli R. 2012. The treatment of jaundice with medicinal plants in indigenous communities of the
Sub-Himalayan region of Uttarakhand, India. J. Ethnopharmacol., 143(1):262291.

Van Acker S, van den Berg D, Tromp M, Griffioen D, van Bennekom W, van der Vijgh W, Bast A. 1996. Structural aspects of antioxidant activity of flavonoids. Free. Radic. Biol. Med., 20(3):331-342.

Yang Q, Liu T, Kuklina E, Flanders W, Hong Y, Gillespie C, Chang M, Gwinn M, Dowling N, Khoury M, Hu F. 2011. Sodium and potassium intake and mortality among US adults: prospective data from the Third National Health and Nutrition Examination Survey. Arch. Intern. Med., 171(13): 1183-1191.

Yayi-ladekan E, Kpoviessi D, Gbaguidi F, Kpadonou-kpoviessi B, Gbenou J, Jolivalt C, Moudachirou M, Accrombessi G, Quetin-leclercq J. 2011. Variation diurne de la composition chimique et influence sur les propriétés antimicrobiennes de l'huile essentielle d'OcimumcanumSims cultivé au Bénin. Int. J. Biol. Chem. Sci., 5(4): 1462-1475. 